\section{PREVENTIVE DENTISTRY}

\section{A prospective study of a caries prevention program in pregnant women and their children five and six years of age}

Gomez SS, Weber AA et al. J Dent Child 2001; 68: 191-195

This programme led to significant long-term reduction of caries in children.

Although fluoride has led to a decline in caries, it still affects many children. Early colonization with $S$. mutans relates to more caries, and preventive programmes have achieved reduction of this organism. The present study compares controls (C) with mothers given a specified preventive programme (PDP) in Chile, and their children.

All subjects drank water originally at $1 \mathrm{ppm} \mathrm{F}^{-}$, which was reduced to $0.6 \mathrm{ppm}$ for the last 2 years. The PDP emphasized oral hygiene and dietary control of caries, and coincided with prenatal obstetric and postnatal paediatric clinic visits.

In 30 PDP mothers and their 5 yr-old children, mean DMFT was 12.6 and dft was 0.4 ; in $30 \mathrm{C}$ pairs, respective scores were 13.1 (NS) and $1.3(P<0.01)$. Respective figures for 37 PDP mothers and 6 yr-old children were 13.7 and 0.2 , and for $C$ subjects, 13.5 (NS) and $1.4(P<0.01)$.

\section{ENDODONTICS}

\section{Epidemiological evaluation of the outcomes of nonsurgical root canal treatment in a large cohort of insured dental patients Lazarski MP, Walker, WA, Ill et al. J Endodon 2001; 27: 791-796}

Endodontists treated more complex problems with similar success rates to general dental practitioners.

This study examined a large US insurance database regarding the results of root canal treatment with at least 2 yrs' follow-up. Dentists who were not endodontists performed 67\% of 44613 procedures, and endodontists 33\%. Case mix differed significantly ( $\mathrm{P}<0.001)$ for the 2 categories: endodontists treated 12\% anteriors, 19\% premolars and 69\% molars, other dentists respectively 21\%, 32\% and 47\%.

Extraction occurred for 5.9\% of endodontist cases, retreatment for $1.5 \%$ and periradicular surgery for $2.1 \%$; respective figures for other dentists were 5.4\%, 3.0\% and 1.1\%. Endodontists performed retreatment and surgery for nearly half of cases initially treated by other dentists. Where periradicular surgery was by other dentists, the subsequent extraction rate was about 4 times greater.

\section{LOCAL ANAESTHESIA; PAEDIATRIC DENTISTRY}

\section{Reactions of children to maxillary} infiltration and mandibular block injections

Ram D, Peretz B Pediatr Dent 2001; 23: 343-346

Similar reactions were forthcoming for both types of injection.

Local anaesthesia may provoke anxiety in in children and dentists treating them. In this study, 26 children aged 4-6 yrs and 34 aged 7-11, who required LA by infiltration and block, were given these injections in random order at 2 visits by experienced paediatric dentists, using $20 \mathrm{~mm} 30$ gauge needles.

Objective rating of childrens' reactions showed significantly more cried in the younger group with infiltrations than blocks ( 9 v. 3 subjects), and there were 3 who moved hands in the younger group only, but response was otherwise similar. Childrens' self-rating of pain according to injection type did not differ, nor did objective or subjective rating vary according to injection order.

\section{ORAL SURGERY: BEHAVIOURAL SCIENCE}

The role of third molar surgery in the exacerbation of eating disorders Maine M, Goldberg MH J Oral Maxillofac Surg 2001; 59: 1297-1300

Eating disorder patients may relapse after surgical removal of third molars.

In the pretreatment assessment of 97 consecutive patients with eating disorders in a Connecticut hospital, subjects were asked whether they had experienced orthodontic treatment or surgery for wisdom teeth, and whether these or other dental procedures had contributed to their eating disorders in any way. Nine patients aged 14-59 yrs gave a positive response: 5 out of a total 53 anorexic, 2 of 17 bulimic, and 2 of 27 with an unspecified eating disorder.

In the whole sample, 16 had orthodontics and 21 had oral surgery. One of the 9 positive respondents said orthodontics alone had exacerbated the eating disorder, and 8 recalled exacerbation following wisdom tooth surgery. The authors suggest this risk needs attention in the history, examination and treatment decision for such patients. A following discussion by a psychologist points out that there was no control group of non-eating-disordered psychiatric patients. 